\title{
SAM Pointed Domain-Containing Ets Transcription Factor
}

National Cancer Institute

\section{Source}

National Cancer Institute. SAM Pointed Domain-Containing Ets Transcription Factor. NCI

Thesaurus. Code C102920.

SAM pointed domain-containing Ets transcription factor (335 aa, 38 kDa) is encoded by the human SPDEF gene. This protein is involved in transcriptional regulation and may modulate both prostate development and prostate cancer progression. 